\title{
Optimal Decompression Through Multi-parametric Nonlinear Programming *
}

\author{
Le Feng*, Christian R. Gutvik**, Tor A. Johansen* \\ * Department of Engineering Cybernetics, Norwegian University of \\ Science and Technology, \\ email:\{feng.le,tor.arne.johansen\}@itk.ntnu.no. \\ ** Department of Circulation and Medical Imaging, Norwegian \\ University of Science and Technology, email:christian.gutvik@ntnu.no.
}

\begin{abstract}
Recently, a comprehensive dynamic mathematical model named Copernicus has been established to discover the mechanism of the vascular bubble formation and growth during and after decompression from a dive. The model uses Venous Gas Emboli (VGE) as a measurement and connects it to the risk of severe Decompression Sickness (DCS). Being validated by a series of diving tests, Copernicus model is believed to be suitable and efficient to predict DCS hence generate optimal decompression profiles for the divers. This paper is based on the Copernicus model and presents a nonlinear model predictive control approach, where multi-parametric nonlinear programming is used to construct an explicit solution for the ease of implementation on a typical low-cost diving computer.
\end{abstract}

Keywords: decompression sickness; multi-parametric nonlinear programming; dynamic decompression model.

\section{INTRODUCTION}

DCS is best known as an injury that affects ascending divers who have breathed gas which is at a higher pressure than the surface pressure due to the pressure of the surrounding water. To prevent DCS and ensure safety many decompression procedures are proposed based on the principles described in Boycott et al. [1908]. The idea is to set up a sufficiently large gradient for gas elimination, which however raises another question - how close to optimal in terms of total decompression time will a decompression procedure be?

To answer this question one needs first an indicator of DCS. Traditional methods tend to use DCS symptoms as the indicator, which can often be summed up by "the bends", "the chokes", "the staggers", and "the tingles" representing the musculoskeletal, pulmonary, inner ear, and skin/central nervous system involvement seen. However there are some ethical concerns provoking DCS on test subjects and the symptomatology of DCS is diffuse. Gutvik and Brubakk [2009] proposed that VGE can also be used as a reliable and sensitive DCS indicator. This has greatly improved the observability of DCS because VGE can be measured using ultrasonic imaging or Doppler.

After having had a DCS indicator one can construct a model to describe the mechanism of how the indicator is affected by different dives and different decompression procedures. A traditional model was proposed in Hills

\footnotetext{
* This work has been supported by UWATEC AG, Switzerland, by The Research Council of Norway and the Norwegian Petroleum Directorate, Norsk Hydro, Esso Norge and Statoil under the "Dive contingency contract" (no 4600002328) with Norwegian Underwater Intervention (NUI).
}

[1970] using DCS symptoms as the indicator. Many decompression procedures are proposed based on this type of model. In Gutvik and Brubakk [2009] a comprehensive dynamic mathematical model (Copernicus) was proposed using VGE as the DCS indicator. An advantage is that this model requires fewer dives and less strenuous protocols to obtain statistically sufficient data (Eftedal et al. [2007]). And in a later test (Gutvik et al. [2009a]) it is also shown that existing decompression algorithms (standard diving tables and protocols) are not consistent in terms of controlling the VGE formation and consequently the risk of DCS.

In this paper, with the Copernicus model, we numerically optimize the decompression procedure by minimizing the total ascend time subject to constraints on the peak decompression stress. Theoretically, solving such an optimal control problem generates a continuous time-depth profile which is not only practically impossible for a diver to follow but also computationally inefficient for later implementation in a low-cost diving computer. Hence two input parameterization methods are studied. The input parameterization methods not only simplify the optimization problem but also reduce the solution space.

Another important issue studied in this paper is on practical implementation in a low-cost diving computer. There are two main obstacles which will be introduced as follows.

The first obstacle is uncertainty, which includes uncertainty within the diver and uncertainty in the environment, e.g., the diver's physiological state change during diving and decompression, pressure, blood perfusion, etc. Another fact is that the diver can never follow the optimal decompression profile perfectly. All of these uncertainty 
may render the decompression profile obtained earlier not optimal anymore during the decompression. A possible way to overcome this obstacle is to solve the optimal control problem in a receding horizon fashion. That is, the dynamic model is updated based on present measurements, and the decompression profile is re-optimized at regular intervals in a diving computer.

The other obstacle is the limited CPU capacity and power consumption requirements for a typical low-cost diving computer. The real-time nonlinear optimization and the receding horizon algorithm requires a large amount of floating point numerical computations per re-optimization. For safety reasons the convergence and correctness of the numerical optimization result needs to be verified. These are considered prohibitive for practical implementation (Feng et al. [2009]). However, the approximate explicit solution of nonlinear constrained optimization using multi-parametric nonlinear programming (mp-NLP) (Grancharova et al. [2007], Johansen [2004]) is expected to be very well suited. Rather than re-computing numerically the optimal solution at each sampling instant based on updated physiological state information, the solution is simply obtained by evaluating a piecewise (non)linear approximation to the optimal solution function. Since such piecewise (non)linear function representation can be precomputed off-line and stored in memory on the diving computer, the computational load on the diving computer will be dramatically relaxed. Moreover, since the solution function is pre-computed, it is easier to verify the correctness and will benefit in the safety-critical applications such as a diving computer.

\section{BRIEF MODEL DESCRIPTION}

The dynamic model introduced in Gutvik and Brubakk [2009] and Gutvik et al. [2009a] is briefly presented here for the ease of reading. The diver can be regarded as a dynamic system where the state vector $x$ is influenced by the input vector $u$.

$$
\begin{aligned}
& \dot{x}=f(x, u) \\
& y=h_{m}(x, u)
\end{aligned}
$$

where

$$
\begin{aligned}
& f(x, u)= \\
& {\left[\begin{array}{c}
\frac{\frac{D \alpha_{b}}{h}\left(x_{2 i}-x_{2 n_{t}+1}-\frac{2 \gamma}{x_{2 i-1}}+P_{\text {meta }}+\frac{c_{s}}{x_{2 i-1}^{3}}\right)-\frac{x_{2 i-1}}{3} u_{1}}{x_{2 n_{t}+1}+\frac{4 \gamma}{3 x_{2 i-1}}-P_{\text {meta }}} \\
\varepsilon_{\tau, i} \frac{\alpha_{b}}{\alpha_{t, i}}\left(u_{2} x_{2 n_{t}+1}-x_{2 i}\right) u_{2+i}+\dot{p}_{r, i}(x) \\
\vdots \\
\vdots \\
u_{1}
\end{array}\right]}
\end{aligned}
$$$$
h_{m}(x, u)=\frac{4 \pi}{3} \delta k_{m} \sum_{i=1}^{n_{t}} r_{i}^{3} V_{i} \omega_{i}-V_{0},
$$$$
x=\left[r_{1}, p_{t, 1}, r_{2}, p_{t, 2}, \ldots, r_{n_{t}}, p_{t, n_{t}}, P_{a m b}\right]^{T},
$$$$
u=\left[\dot{P}_{a m b}, f_{N_{2}}, \omega_{1}, \omega_{2}\right]^{T} \text {, }
$$

$$
\begin{aligned}
\dot{p}_{r_{i}}(x) & =-\frac{4 \pi D \alpha_{b} \delta}{h \alpha_{t, i}} x_{2 i-1}^{2}\left(x_{2 i}-x_{2 n_{t}+1}-\frac{2 \gamma}{x_{2 i-1}}\right. \\
& \left.+P_{m e t a}+c_{s} \frac{1}{x_{2 i-1}^{3}}\right)
\end{aligned}
$$

The model is constituted of $n_{t}$ tissues. Theoretically $n_{t}$ can be chosen as large as we want therefore the model can approximate a human body as precisely as needed. On the other hand it's convenient to keep $n_{t}$ as small as possible considering the size of the resulting optimization problem. In this paper we use two tissues $(i=\{1,2\})$, the muscles and fat tissues, which represent the dynamics of fast and slow tissues, respectively Gutvik et al. [2009a,b]. The nomenclature is presented as below.

\section{OPTIMAL DECOMPRESSION PROBLEM}

\subsection{Parameterization of the decompression profile}

The main purpose of the optimal decompression is to ascend a diver as fast as possible while the size of bubbles in the pulmonary (output $y$, also referred to as the decompression stress) does not exceed a pre-defined threshold. A stepwise decompression formulation is normally used, illustrated as in Figure 1.

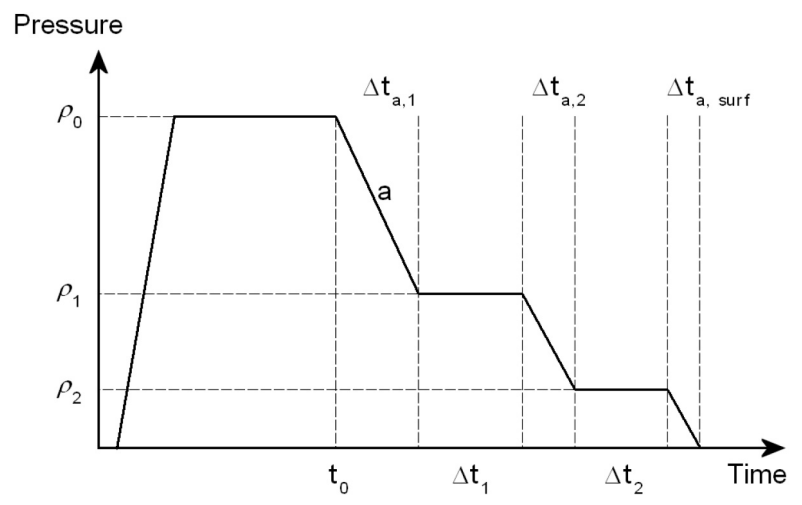

Fig. 1. Stepwise decompression profile parameterization (Tøndel et al. [2005])

A fixed number of stopping depths $\left(\rho_{1}, \rho_{2}, \ldots, \rho_{n_{s}}\right)$ are defined. Assuming the ascending speed of the diver $(a)$ is constant, the time for ascending $\Delta t_{a, 1}+\Delta t_{a, 2}+\ldots+$ $\Delta t_{a, \text { surf }}$ is a constant. Suppose $\Delta t_{1}, \Delta t_{2}, \ldots, \Delta t_{n_{s}}$ are the stopping times on each stopping depth. Hence the goal of optimal decompression is to

$$
\min \sum_{i=1}^{n_{s}} \Delta t_{i}
$$

With such parameterization there are $n_{s}$ parameters to be optimized subject to $\Delta t_{i} \geqslant 0$. However in some cases, e.g., a long deep dive, there shall be quite many stopping depths to safely ascend a diver, which means $n_{s}$ can still be large and the computational effort can still be huge. In addition, this parameterization tends to give a "flat minimum" with associated numerical challenges. This motivated another parameterization method used in Gutvik et al. [2009b] and studied in Feng et al. [2009], illustrated in Figure 2. 


\begin{tabular}{|c|c|c|c|}
\hline Param. & Description & Value & Unit \\
\hline$n_{t}$ & Number of tissues. & 2 & - \\
\hline$D$ & Bubble barrier diffusivity. & 20 & $\mu m^{2} / \mathrm{min}$ \\
\hline$\alpha_{b}$ & Blood solubility. & 0.0158 & $\mathrm{msw}^{-1}$ \\
\hline$h$ & Bubble barrier thickness. & 0.1 & $\mu m$ \\
\hline$\gamma$ & Surface tension. & 17.890 & $\mu m \mathrm{msw}$ \\
\hline$P_{\text {meta }}$ & Partial pressure of the metabolic gases. & 0.1773 & atm \\
\hline$c_{s}$ & Coefficient. & 0.9479 & $\mu m^{3}$ atm \\
\hline$\varepsilon_{\tau, 1}$ & Time constant correction of muscles. & 0.2868 & - \\
\hline$\varepsilon_{\tau, 2}$ & Time constant correction of fat. & 0.8115 & - \\
\hline$\alpha_{t, 1}$ & Tissue solubility of muscles. & 0.0278 & $\operatorname{atm}^{-1}$ \\
\hline$\alpha_{t, 2}$ & Tissue solubility of fat. & 0.0640 & $\operatorname{atm}^{-1}$ \\
\hline$\delta$ & Bubble nuclei density. & $5 \cdot 10^{-7}$ & $\# / \mu m^{3}$ \\
\hline$k_{m}$ & Measurement gain. & 200 & - \\
\hline$V_{0}$ & Dead volume for detection. & 0.005 & \#bubbles $/ \mathrm{cm}^{2} / \mathrm{min}$ \\
\hline$V_{1}$ & Tissues volume of muscles. & 28.4 & $\mathrm{dm}^{3}$ \\
\hline$V_{2}$ & Tissues volume of fat. & 11.7 & $\mathrm{dm}^{3}$ \\
\hline$r_{i}$ & Bubble radius in tissue $i$. & & $\mu m$ \\
\hline$p_{t, i}$ & Gas tension in tissue $i$ & & ATA \\
\hline$P_{a m b}$ & Ambient pressure. & & msw \\
\hline$\dot{P}_{a m b}$ & Rate of descent. & & $\mathrm{msw} / \mathrm{min}$ \\
\hline$f_{N_{2}}$ & Fraction of nitrogen in inspired gas. & 0.78 & - \\
\hline$\omega_{1, \text { dive }}$ & Blood perfusion of muscles (dive). & 0.16 & $\mathrm{~L} / \mathrm{min} / \mathrm{dm}^{3}$ \\
\hline$\omega_{1, \operatorname{surf}}$ & Blood perfusion of muscles (surface). & 0.091 & $\mathrm{~L} / \mathrm{min} / \mathrm{dm}^{3}$ \\
\hline$\omega_{2, \text { dive }}$ & Blood perfusion of fat (dive). & 0.047 & $\mathrm{~L} / \mathrm{min} / \mathrm{dm}^{3}$ \\
\hline$\omega_{2, \text { surf }}$ & Blood perfusion of fat (surface). & 0.043 & $\mathrm{~L} / \mathrm{min} / \mathrm{dm}^{3}$ \\
\hline$y$ & Measurement of the number of bubbles in the pulmonary artery. & & \#bubbles $/ \mathrm{cm}^{2} / \mathrm{min}$ \\
\hline
\end{tabular}

Table 1. Nomenclature

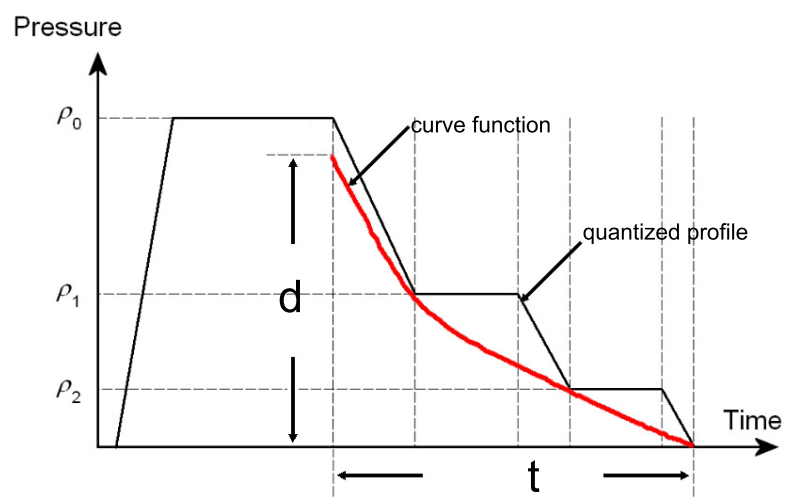

Fig. 2. Curve decompression profile parameterization (Feng et al. [2009])

The idea is to first define a curve function with two parameters $d$ and $t$ (illustrated in Figure 2). For a given $d$ and $t$, the curve can be quantized and generate the stepwise formulation and also a total ascend time according to stopping depths $\left(\rho_{1}, \rho_{2}, \ldots, \rho_{n_{s}}\right)$. Then instead of optimizing $n_{s}$ parameters in the original formulation, now only two parameters need to be optimized. Obviously this parameterization and quantization method leads to a simplified optimization problem but may also introduces some conservatism, that is, the total ascending time $(t)$ may be longer and/or the stress threshold may be exceeded. The benefit and performance loss of this parameterization method was studied by simulations in Feng et al. [2009].

\subsection{Constraints}

The nonlinear constraints setup is in a predictive manner. This is because generally the bubbles in the pulmonary artery are first compressed due to the increasing ambient pressure while the diver is descending, and start growing in size after the maximum depth is reached. The growing speed depends on several factors, such as pressure, diver's heart rate, and decompression profile. We may not know in advance at which time the maximum decompression stress is reached. Generally the bubbles will reach their peak sizes sometime after the diver arrives the surface. Therefore to avoid violating a predefined threshold on the VGE peak, the output $y$ (decompression stress) at not only current time but also during a prediction horizon shall be considered and kept under the threshold. Mathematically, the nonlinear constraints can be written as

$$
H\left(z, x_{0}, n_{k}\right)=\left[\begin{array}{c}
y\left(x_{1}\right)-y_{\text {limit }} \\
y\left(x_{2}\right)-y_{\text {limit }} \\
\vdots \\
y\left(x_{n_{k}}\right)-y_{\text {limit }}
\end{array}\right] \leqslant 0 .
$$

where $x_{0}$ is the current state, the future state $x_{i}$ is predicted through numerical integration of the model from $x_{0}$ and $i \in\left\{1,2, \ldots, n_{k}\right\}$ are the discretization times. Please note that $n_{k}$ shall be large enough to allow the bubbles to reach their peak sizes.

\subsection{Optimization problem}

The optimal decompression problem using curve parameterization is given as follows:

$$
\min _{t, d} \sum_{i=1}^{n_{s}} \Delta t_{i}
$$

s.t.

$$
-q_{t}(t, d) \leqslant 0
$$

$H\left(q_{t}(t, d), x_{0}, n_{k}\right) \leqslant 0$,

System dynamics in (1), 
where $z=\left[\Delta t_{1}, \Delta t_{2}, \ldots, \Delta t_{n_{s}}\right]^{T}=q_{t}(t, d)$ is the curve quantization function illustrated in Fig. 2.

Considering the uncertainty, the optimization problem needs to be solved in a receding horizon control manner. The diving computer first measures the ambient pressure $P_{a m b}$ and heart rate of the diver; then estimates a current state based on this measurement, manual input, and the mathematical model; uses this information to generate an optimal decompression profile for the diver. The diver tries to follow the command he received and ascend to a stopping depth as suggested (which might not be very accurate). After which the diving computer measures a new ambient pressure $P_{a m b}$ and heart rate and solves the optimization problem repetitively at the new time step.

\section{PIECEWISE NONLINEAR EXPLICIT SOLUTION}

\subsection{Introduction to multi-parametric nonlinear programming}

In order to obtain a piecewise nonlinear explicit solution function out of the optimization problem (4), a brief introduction to multi-parametric nonlinear programming (Fiacco [1983]) is presented here for the ease of reading.

Basically, in RHC optimization it is regarded as a natural extension of explicit solutions derived for linear input and state constrained RHC and linear quadratic regulation (Bemporad et al. [2000], Bemporad and Filippi [2001]). The main difference is that in linear cases the exact state feedback solution takes the form of a piecewise linear (PWL) function which can be pre-computed off-line using multi-parametric quadratic programming (mp-QP) algorithms (Bemporad et al. [2000]), while in the nonlinear cases no exact state feedback solution can be finitely represented in general but approximately described using mp-NLP. Recently in Johansen [2004] mp-NLP is used to extend from linear RHC (Johansen and Grancharova [2003]) to nonlinear constrained RHC problems. The main differences are related to replacing several quadratic programs (QP) sub-problems with nonlinear programming (NLP) sub-problems. The algorithm in Johansen and Grancharova [2003], Johansen [2004] has the advantage that a computationally favorable PWL approximation is implemented via a binary search tree.

The algorithms to find a piecewise linear and nonlinear explicit solution are discussed in detail in Grancharova et al. [2007], Grancharova and Johansen [2009]. Basically these approaches differs from each other in the approximation functions. The piecewise nonlinear approximation function has more powerful approximation ability but also leads to more computational demand compared to the piecewise linear approximation function. In both approaches the solution is found by partitioning the parameter space into a set of hypercubes, and giving a piecewise (non)linear explicit approximation of the NLP in each hypercube. Putting these mp-NLP solutions together gives an approximate solution of the NLP as a function of the parameter. This means that the main effort of solving the optimization problem is moved off-line. The on-line effort is then reduced to evaluating a piecewise function, which can be implemented efficiently using a binary search tree (Johansen [2004]).

\subsection{Piecewise nonlinear explicit solution to the optimal decompression problem}

Looking back at the optimal decompression problem described in this paper, the re-optimization requires computational effort which is considered hard to implement, especially on a typical low-cost diving computer. In the light of multi-parametric nonlinear programming, an explicit solution can be obtained and is suitable in this case. Considering the requirements and complexity of the decompression problem, in this paper we use a piecewise quadratic function to approximate the exact optimal solution.

It can be defined that $\theta=[t, d] \in \mathbb{R}^{2}$ in (4) is a vector of decision variables, and the time-varying state $x_{0}$ should be considered a parameter vector to the optimization problem. Using barrier functions as in Feng et al. [2009] (4) can be considered an mp-NLP, which means that the optimal solution should be found for a given range of the parameter values. We seek an explicit approximate representation of the solution as a function of these parameters. The algorithm can be summarized as follows.

For a given hypercube $X_{0}$,

1. Solve the optimization problem (4) when $x_{0}$ equals to each and every vertex of $X_{0}$;

2. If all the optimizations are feasible, goto step 3 . Otherwise check if the size of $X_{0}$ is too small. If yes, mark $X_{0}$ as explored and infeasible and return. If no goto step 5;

3. Compute a quadratic function to best approximate the optimal solutions obtained in step 1 ;

4. Estimate the approximation error in $X_{0}$. If the error is under a pre-defined error bound, mark $X_{0}$ as explored and feasible and return, else goto step 5 .

5. Split $X_{0}$ following some heuristic rules described in Johansen [2004], mark both unexplored and call the procedure recursively for each of them.

Recursively running this algorithm will partition the initial parameter space into a set of hypercubes, and give an acceptable (by defining an error bound) piecewise quadratic approximation of each hypercube. Putting these piecewise solutions together gives an approximate solution to the NLP as a function of the parameter, i.e.,

$\theta\left(x_{0}\right)=\left[\begin{array}{c}t \\ d\end{array}\right]=K_{2, j} x_{0}^{T} \cdot x_{0}+K_{1, j} x_{0}+K_{0, j}, \quad$ if $\quad x_{0} \in R_{j}$

where $x_{0} \cdot x_{0}$ means dot product, $R_{j}$ is the $j_{\text {th }}$ hypercube, $K_{2, j}, K_{1, j}$ and $K_{0, j}$ are approximate parameters according to the hypercube $R_{j}$.

\section{SIMULATION RESULTS}

In this paper we have restricted our attention to decompression profiles for depths from 0 to $50 \mathrm{~m}$ and the total decompression time less than 64 minutes, with a stepwise decompression trajectory with stops at every $3 \mathrm{~m}$ from 0 to $15 \mathrm{~m}$. That is, for dives to depth from $50 \mathrm{~m}$ to $15 \mathrm{~m}, 5$ stops are considered. For dives to depth between 15 and 12 m, 4 stops are considered, and so on. All these parameters can be easily modified in the algorithm, depending on what kind of dives are of interest. 
Based on the range of the dives defined above we can simulate the Copernicus model and find the 5-dimensional state space that interests us. Please note that the bubbles start growing during bottom time and the decompression phase. Therefore the bounds on the bubble sizes $\left(r_{1}\right.$ and $\left.r_{2}\right)$ as well as the gas tension $\left(P_{t, 1}\right.$ and $\left.P_{t, 2}\right)$ should be chosen carefully. Too small bounds may render the explicit solution not applicable in some cases while too large bounds can lead to a very long computational time to fully explore the whole state space. In this paper the state space inside which the explicit solution is computed is given below.

$$
\begin{aligned}
0.2550 \mu \mathrm{m} & <r_{1}<4.3473 \mu \mathrm{m} \\
11.0971 \mathrm{ATA} & <P_{t, 1}<24.3381 \mathrm{ATA} \\
0.2483 \mu \mathrm{m} & <r_{2}<2.8658 \mu \mathrm{m} \\
9.1156 \mathrm{ATA} & <P_{t, 2}<17.6887 \mathrm{ATA} \\
11.0000 \mathrm{msw} & <P_{a m b}<60.0000 \mathrm{msw}
\end{aligned} .
$$

The model is integrated at a varying integration step. During decompression stop, the integration step is the stopping time divided by 30 . Integration step during accent phases between decompression stops is $\frac{1}{9}$ minute. The prediction horizon after ascending to the surface is set to 900 minutes (until the estimated stress reaches its maximum).

The explicit solutions is obtained off-line which takes 129 hours with 4591 regions. All the computations are done on an Intel Due 2.53G desktop where each NLP is solved in Matlab with the TOMLAB 'conSolve' algorithm using a Quasi-Newton method BFGS. We use a binary search tree to locate the region corresponding to the state. In this case at most 20 arithmetic operations per update are needed. The arithmetic operation can be implemented with fixed point arithmetics such that floating point capability of the diving computer is not needed.

Using the explicit solution two decompression scenarios are simulated. We assume the diver can ascend at a fixed speed $a$, but may have problem stopping exactly as long as suggested by the diving computer. Because of the uncertainty on the stopping time, an updated decompression profile must be produced to the diver.

In the first scenario we assume the diver rounds off the suggestion from the diving computer for the ease of timing. The result is given in Table 2 and plotted in Figure 3. The first column of the table is current depth; the second column is the optimal solution given by the proposed method before the stop; the third column is the suggested stopping time at current depth while the forth column is the real stopping time at current depth; the fifth column is the updated optimal solution after the recent stop. In Figure 3 the sub-figure on the top gives the time-depth decompression profile while the one on the bottom is the output $y$ (solid line, measurement of the number of bubbles in the pulmonary artery). The dotted line in the bottom sub-figure is the stress threshold which is designed not to be violated during simulation.

The total decompression time is 63 minutes, which is $11.14 \%$ longer than the optimal solution obtained at 50 $\mathrm{m}$ depth. This is because of the uncertainty and also the curve approximation method.
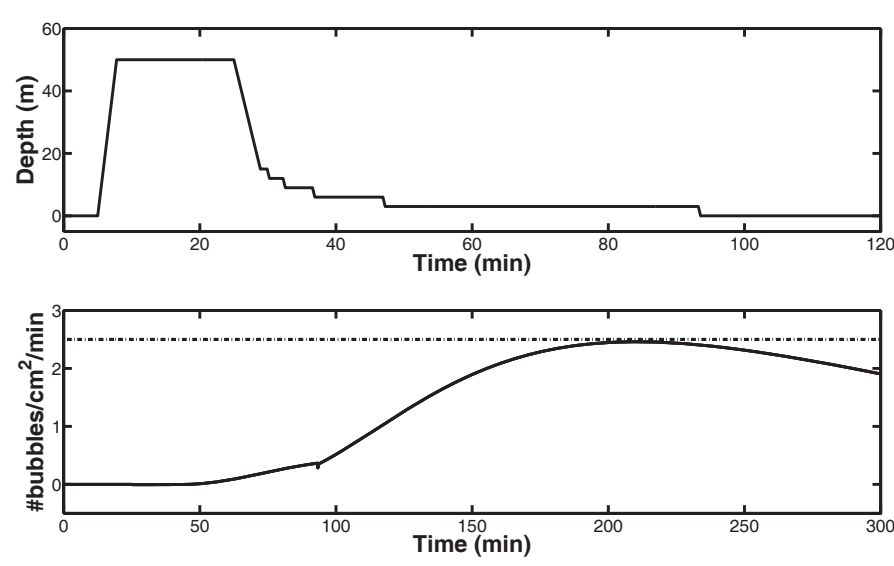

Fig. 3. Decompression scenario 1 of the diving schedule $[50,20,95,0.78]$

In the second scenario we assume the diver is more careless. During the simulation the suggestion from the computer is only taken as a reference. The real stopping time varies much from the optimal one. So it's a more harsh test of the explicit solution. The result is given in Table 3 and plotted in Figure 4.
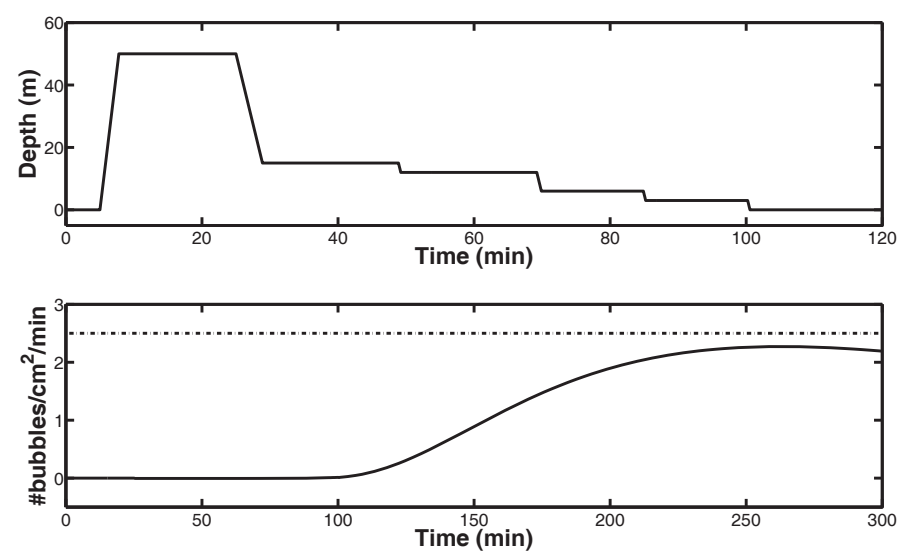

Fig. 4. Decompression scenario 2 of the diving schedule $[50,20,95,0.78]$

The total decompression time is 70 minutes.

From the simulation results we can see that the proposed method gives updated optimal decompression profiles although there's some uncertainty.

\section{CONCLUSION}

In this paper we applied a new dynamic model on bubble formulation and evolution in human body. The model is based on Venous Gas Emboli (VGE) rather than decompression sickness (DCS). Based on this model the decompression problem is formulated as a nonlinear optimal control problem, i.e., minimizing the total decompression time subject to safety constraints. The explicit solution is obtained using piecewise approximation method and multi-parametric programming. Simulation is carried on a two tissue model and the results are promising.

\section{REFERENCES}

A. Bemporad and C. Filippi. Suboptimal explicit MPC via approximate quadratic programming. Proceedings 


\begin{tabular}{rrrrr}
\hline Depth & Optimal solution before the stop & Suggested & Real & Optimal solution after the stop \\
\hline 50 & & & 20.0000 & {$[0.6004,3.4440,8.8124,16.7055,27.1235]$} \\
15 & {$[0.6004,3.4440,8.8124,16.7055,27.1235]$} & 0.6004 & 1.0000 & {$[0.0000,2.1956,7.6781,16.6056,28.9779]$} \\
12 & {$[0.0000,2.1956,7.6781,16.6056,28.9779]$} & 2.1956 & 2.0000 & {$[0.0000,0.0000,4.2747,16.6338,37.4344]$} \\
9 & {$[0.0000,0.0000,4.2747,16.6338,37.4344]$} & 4.2747 & 4.0000 & {$[0.0000,0.0000,0.0000,10.8001,46.3912]$} \\
6 & {$[0.0000,0.0000,0.0000,10.8001,46.3912]$} & 10.8001 & 10.0000 & {$[0.0000,0.0000,0.0000,00.0000,46.2025]$} \\
3 & {$[0.0000,0.0000,0.0000,00.0000,46.2025]$} & 46.2025 & 46.0000 & \\
\hline
\end{tabular}

Table 2. Decompression scenario 1 with uncertainty from diver. Dive schedule: $[50,20,95,0.78]$

\begin{tabular}{rrrrr}
\hline Depth & Optimal solution before the stop & Suggested & Real & Optimal solution after the stop \\
\hline 50 & & & 20.0000 & {$[0.6004,3.4440,8.8124,16.7055,27.1235]$} \\
15 & {$[0.6004,3.4440,8.8124,16.7055,27.1235]$} & 0.6004 & 20.0000 & {$[0.0000,1.7460,6.1611,13.3707,23.3748]$} \\
12 & {$[0.0000,1.7460,6.1611,13.3707,23.3748]$} & 1.7460 & 20.0000 & {$[0.0000,0.0000,2.1692,08.3724,18.7866]$} \\
9 & {$[0.0000,0.0000,2.1692,08.3724,18.7866]$} & 2.1692 & 0.0000 & {$[0.0000,0.0000,0.0000,06.3185,25.0768]$} \\
6 & {$[0.0000,0.0000,0.0000,06.3185,25.0768]$} & 6.3185 & 15.0000 & {$[0.0000,0.0000,0.0000,00.0000,12.8902]$} \\
3 & {$[0.0000,0.0000,0.0000,00.0000,12.8902]$} & 12.8902 & 15.0000 & \\
\hline
\end{tabular}

Table 3. Decompression scenario 2 with uncertainty from diver. Dive schedule: [50, 20, 95, 0.78]

of the IEEE conference on decision and control, pages 4851-4856, 2001.

A. Bemporad, M. Morari, V. Dua, and Pistikopoulos E. N. The explicit solution of model predictive control via multiparametric quadratic programming. American Control Conference, 2000.

A. E. Boycott, C. C. Damant, and J. S. Haldane. The prevention of compressed - air illness. Journal of Hygiene, 8(342-443), 1908.

O. S. Eftedal, S. Lydersen, and A. O. Brubakk. The relationship between venous gas bubbles and adverse effects of decompression after air dives. Undersea $\&$ hyperbaric medicine: journal of the Undersea and Hyperbaric Medical Society, 34(2):99-105, 2007.

L. Feng, C. R. Gutvik, T. A. Johansen, and D. Sui. Barrier function nonlinear optimization for optimal decompression of divers. Proceeding of 48 th IEEE Conference of Decision and Control, 2009.

A. Fiacco. Introduction to sensitivity and stability analysis in nonlinear programming. Elsevier, 1983. ISBN 9780080956718 .

A. Grancharova and T. A. Johansen. Computation, approximation and stability of explicit feedback minmax nonlinear model predictive control. Automatica, 45 (5):1134-1143, 2009.

A. Grancharova, T. A. Johansen, and P. Tøndel. Computational aspects of approximate explicit nonlinear model predictive control. In R. Findeisen, F. Allgöwer, and Biegler L., editors, Assessment and Future Directions of Nonlinear Model Predictive Control, volume 358 of Lecture Notes in Control and Information Sciences, pages 181-192. Springer Berlin / Heidelberg, 2007.

C. R. Gutvik and A. O. Brubakk. A dynamic 2-phase model for vascular bubble formation during decompression of divers. IEEE Transactions Biomedical Engineering, 2009.

C. R. Gutvik, R. G. Dunford, Z. Dujic, and A. O. Brubakk. Parameter estimation of the copernicus decompression model using non-linear optimization. Medical and Biological Engieering and Computing, 2009a.

C. R. Gutvik, T. A. Johansen, and A. O. Brubakk. Nonlinear optimization of a 2-phase decompression model and an approximate, explicit solution. In preparation,
$2009 \mathrm{~b}$

B. A. Hills. Vital issues in computing decompression schedules from fundamentals i. critical supersaturation versus phrase equilibration. Int J Biometeror, 14(2): 111-128, 1970.

T. A. Johansen. Approximate explicit receding horizon control of constrained nonlinear systems. Automatica, 40:293-300, 2004.

T. A. Johansen and A. Grancharova. Approximate explicit model predictive control via orthogonal search tree. IEEE Transactions on Automatic Control, 48:810-815, 2003

Petter Tøndel, C. R. Gutvik, and T. A. Johansen. Optimal decompression - diving computer implementation aspects of an explicit solution. Technical report, Department of Engineering Cybernetics, Norwegian University of Science and Technology, 2005. 